\title{
Phase Response Calibration of a Distributed Antenna Array for Satellite Acquisition
}

\author{
Alberto Antón, Isabel García-Rojo, Alejandro Girón, Eva Morales, and Ramón Martínez
}

\begin{abstract}
A distributed antenna array system, named Spanish acronym for fast acquisition of satellites system (SARAS), has been designed as an acquisition aid to parabolic telemetry, tracking, and command antennas when tracking satellites in launch and early orbit phase or during critical maneuvers. The system relies on digital signal processing, based on the array changing phase response to incoming signals as a function of their direction of arrival. One critical aspect for proper performance of the system is to establish a reliable model of this phase response, termed the array manifold, prior to and during satellite acquisition, through novel calibration techniques that are the focus of this letter.
\end{abstract}

Index Terms-Antenna arrays, array manifold, calibration, digital signal processing, direction of arrival (DoA) estimation, ground stations, launchers, launch and early orbit phase (LEOP), telemetry, tracking, and command (TT\&C), satellites.

\section{INTRODUCTION}

G ROUND stations aimed to receive or transmit data from/to orbiting satellites and other spatial vehicles need to know their angular location with great precision and accuracy. Indeed, large antennas in the ground segment are characterized for having a narrow beam and, thus, a high gain. Such narrow beams become a problem in the initial phases of satellite acquisition, especially when dealing with launch and early orbit phase. As the angular uncertainty window during this phase can reach values of up to $\pm 1.5^{\circ}$, it can take a long time before the ground station acquires the satellite, causing data losses and obviously potential inability to operate the spacecraft.

It is thus in this context that an innovative array-based solution, termed Spanish acronym for fast acquisition of satellites system (SARAS), has been proposed [1] as an acquisition-aid system for satellite communications. The array is composed of eight radiating elements operating in S-band $(2.2-2.3 \mathrm{GHz})$, uniformly distributed in the rim of a 15 -m telemetry, tracking, and command (TT\&C) dish, and has the following key characteristics:

1) high white noise robustness;

2) fast acquisition through electronic steering;
3) tracking and prediction of trajectories;

4) interferer discrimination.

The inclusion of more array elements increases the robustness against white noise, as the array gain is enhanced. However, simulations of the system have shown that improvements beyond eight elements are residual and do not justify the extra hardware and installation costs.

However, the direction of arrival (DoA) estimation process, which uses statistical information from the received samples, relies on assumptions regarding the phase response model of the array. Errors in such modeling lead to quick degradation of performance.

Thus, establishing the functional relationship between phase response and signal DoA is a critical aspect of the system, and is grouped into what is generally known as array calibration. Calibration techniques in SARAS were already introduced in [2], but will be expanded here to offer a complete view of the proposed solution, along with simulations and some considerations regarding frequency-dependent errors.

As will be detailed in the next sections, the proposed calibration scheme is more robust than classical solutions because it relies on a dual modeling paradigm, wherein calibration algorithms are applied prior to satellite acquisition (offline calibration) and simultaneously (online calibration). Online calibration is advantageous to compensate dynamic errors, while offline calibration can be used as a filtering tool to eliminate from the online stage all the spurious error sources that do not provide reliable data regarding the phase response of the array.

\section{SARAS CaLibration SCHEME}

\section{A. Introduction}

Phase differences between received signal samples in an array change with the DoA of the satellite to be acquired. Thus, estimating them with statistical averages is a mean of determining the angular location of the desired signal. However, the vector function that relates array phase differences to signal DoA is generally not known a priori and is nonlinear in nature.

The calibration process seeks to infer this vector function, termed the array manifold or the steering vector. However, as is usually the case in nonlinear scenarios, the estimation techniques must be carefully designed to avoid local minima and nonconvergence. Two main procedures can be defined:

1) A nonparametric approach, which seeks to directly obtain the numerical values of the array manifold for a set of steering directions of the array. This set can be made as exhaustive as required, and complemented with interpolation when needed. 


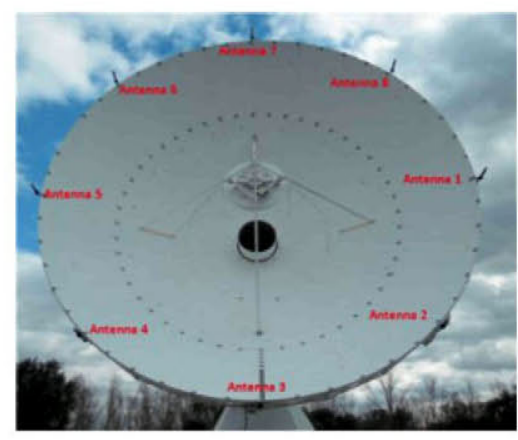

Fig. 1. SARAS system and photogrammetric targets.

2) A parametric approach, where the array manifold is expressed as an equation that depends on a given set of DoA-independent unknowns. If estimated, numerical values can be obtained for any desired DoA.

SARAS scheme presents a combination of both approaches, using the parametric modeling for those calibration errors that are more stable in time, and the nonparametric approach for dynamic perturbations that cannot be easily modeled a priori. Section II-B will explore the first stage in calibration, photogrammetry, which is a parametric technique for the estimation of the geometrical topology of the array.

\section{B. Photogrammetry}

In this parametric calibration, the parameters to be obtained are the Cartesian coordinates of the array elements. To obtain these coordinates, photogrammetry has been considered. Photogrammetry is a technique that extracts three-dimensional (3-D) shapes from a set of two-dimensional pictures, taken with different orientations. With the help of a set of retro-reflective targets placed in the main antenna, the 3-D geometrical shape of the dish and the array sensors can be extracted, thus allowing estimating groups of coordinates and, in the end, calculate a coordinate system that relates the array elements to the steering of the main antenna. High accuracy can be achieved, even with off-the-shelf cameras.

Fig. 1 shows the retro-reflective targets placed in the main dish, the positions of which were estimated during the photogrammetric campaigns. It also shows the eight-array radiating elements that are a part of SARAS system.

Some ideas regarding the required processing algorithms can be found in [3]. Also, it should be noted that similar systems as the one intended can be found in [4] and [5]. However, in those references, the aim is to determine the whole shape of the antenna or reflector dish. A photogrammetric calibration of a phased array is performed in [6].

\section{Offline Calibration}

All the perturbations that are not included in the photogrammetric model, mainly those due to phase offsets in analog components, must be compensated through nonparametric algorithms.

As indicated, offline calibration is performed prior to signal acquisition. This stage uses two frequency-multiplexed carri- ers, one generated by a probe placed in the subreflector of the main TT\&C antenna and, thus, having visibility of all array elements, and another received from a satellite in geostationary orbit. Frequency offsets are set between 100 and $200 \mathrm{kHz}$. Received samples are processed in the following way:

1) A fast Fourier transform (FFT) of the incoming samples is performed, and the frequency bins around the highest peak are selected. These correspond to the probe signal since it has more power than the signal from the satellite.

2) The amplitude and phase offsets of the probe signal are estimated by calculating the frequency array correlation matrix and obtaining its dominant eigenvector [7].

3) The probe signal is filtered out from the incoming samples.

4) The same process is repeated with the carrier from the GEO satellite.

The rationale behind this processing is similar to the one used in astronomy, when using the technique termed same beam interferometry [8]. In any case, this stage only seeks to model phase differences between the RF propagation paths of the probe signal and the ones from a satellite signal coming from broadside with respect to the array system, as will be detailed in Section II-D. The differences are computed as follows:

$$
\Delta \phi_{\text {delta }}=\Delta \phi_{\text {GEO }}-\frac{2 \pi}{\lambda} \mathbf{z}-\Delta \phi_{\text {probe }} .
$$

$\Delta \phi$ is an $N \times 1$ vector containing the phase offsets among array branches for the corresponding signal, obtained from the dominant eigenvector in the array correlation matrix. $N$ is the number of array elements, $\mathbf{z}$ are the Cartesian coordinates as estimated in photogrammetry, and $\lambda$ is the wavelength of the GEO satellite carrier.

\section{Online Calibration}

This stage is performed during signal acquisition, using the same frequency-multiplexed procedure as before. In this case, one of the signals originates from the satellite to be acquired, while the other comes from the subreflector probe.

The problem with offline techniques is that the phase differences they estimate correspond to an array model that may no longer be valid during acquisition, mainly due to instabilities in the phase response of analog components in the array receiving chains. In fact, this was found during test campaigns to be the case with SARAS. The offline calibration models degraded quickly when operating with real satellites, due to unstable ana$\log$ components.

The online calibration clearly overcomes dynamic degradations, while eliminating the need to use highly stable and expensive hardware. It cannot be applied on its own, though, since it requires the use of the subreflector probe signal, which corresponds to a calibration model that differs from the one that can be applied to a satellite signal. These differences come from spurious components, namely:

1) the near-field perturbation of the received signal;

2) the radiation patterns of the antennas, which see the received signal from different directions that do not coincide with the broadside one; 


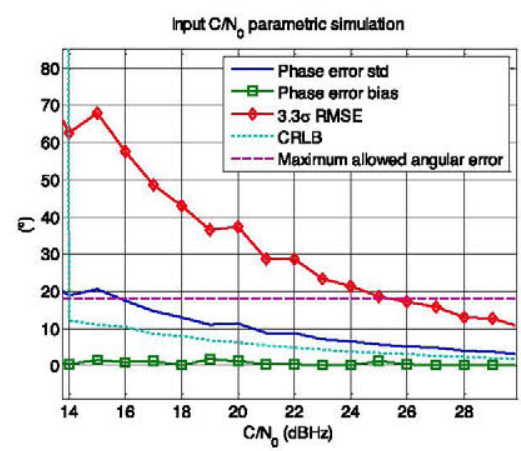

Fig. 2. Eigen performance in the presence of white noise.

3) the different path lengths from the probe to each receiving antenna.

As commented, the offline stage with the GEO satellite has been considered in order to cancel out these spurious components. Of course, the assumption is that they remain approximately constant between both stages.

Finally, the phase estimates from both stages are combined to obtain the final calibration model, which is used by the DoA estimation algorithm

$$
\Delta \phi_{\text {final }}=\Delta \phi_{\text {delta }}+\Delta \phi_{\text {on-line }} .
$$

\section{Simulations}

Monte Carlo simulations have been carried out to assess the validity of the scheme.

First, the effect of noise on the ability of the Eigen algorithm to estimate phase offsets in the array manifold has been simulated, using a nonmoving source. The idea is to reproduce conditions of the offline calibration stage, where a GEO satellite is considered, since this is the case where the lowest C/No (Carrier over Noise floor level) values will be encountered, when compared to the power levels of the subreflector probe beacon. Results are shown in Fig. 2.

Results show the standard deviation (std), the bias, and the root mean square error of the phase response estimates, along with the Cramér-Rao lower bound (CRLB) [9]. A threshold has been set to $18^{\circ}$ since this was found to be an upper limit to obtain acceptable results in DoA estimation. It is clear from the results that $\mathrm{C} / \mathrm{No}$ above $25 \mathrm{dBHz}$ is recommended. The simulation was carried using $10^{5}$ samples for computing statistical averages. Of course, the $\mathrm{C} / \mathrm{N}$ o threshold can be lowered by increasing this amount. On the other hand, increasing the number of samples increases the computing time and, more importantly, nonstationary degradation, i.e., satellite movement, becomes more acute, as explored in the next simulation.

Even though in Fig. 2 it has been assumed that Doppler shift is very low with GEO satellites, even a small angular offset can affect the calibration scheme, especially when processing a large amount of samples. Fig. 3 establishes, for a C/No of 25 $\mathrm{dBHz}$ and $10^{5}$ samples, what is the maximum angular speed that a GEO satellite used for calibration can have.

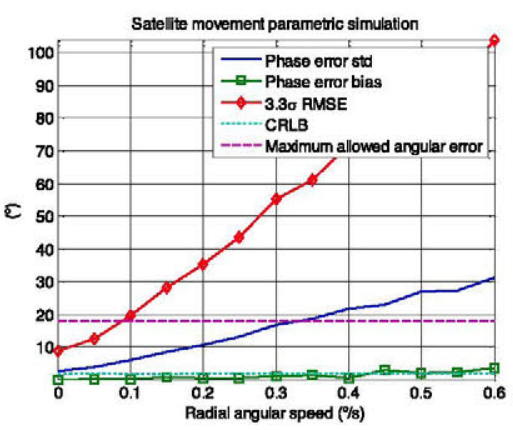

Fig. 3. Eigen performance in the presence of angular drift.

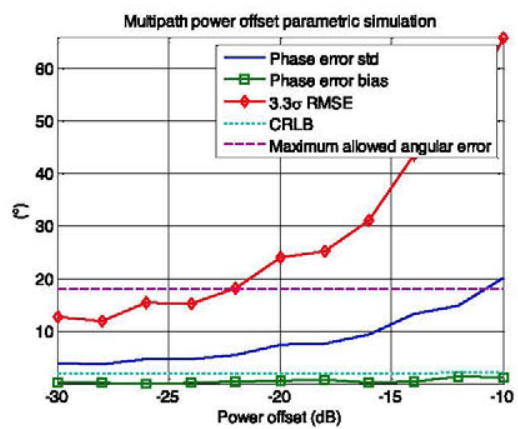

Fig. 4. Eigen performance in the presence of multipath.

Radial speeds greater than $0.1 \%$ would degrade calibration estimates. The requirement becomes more stringent if more samples are processed, with a linear dependence.

The effect of multipath has also been explored in Fig. 4. Multipath does not affect the offline calibration stage when using GEO satellites, due to their high elevations $\left(>5^{\circ}\right)$, but this is no longer the case should a collimation tower be considered instead. This is indeed an upgrade which may be used in future implementations of the system.

Multipath has been modeled as an additional signal, fully correlated with the desired one, with a different array manifold to account for the angular offsets between both signals. Multipath power offsets below $-22 \mathrm{~dB}$ are required. This may not be easily achieved at low elevations $\left(<5^{\circ}\right)$, so this upgrade may require specific algorithms to cancel out the multipath contribution.

\section{FREQUENCY-DEPENDENT ERRORS}

Nonparametric phase estimates can be divided into two components:

1) GEO satellite estimates at a given frequency;

2) dynamic errors at the desired signal frequency.

The sum of both components gives the actual calibration estimate. It is clear that large frequency offsets between both of them will produce uncompensated errors. Unfortunately, the array phase response as a function of frequency is not known. Indeed, there is a known dependence of the form

$$
\Delta \phi \sim \frac{2 \pi}{c} \Delta f \times g(\Delta p, \theta, \varphi) .
$$




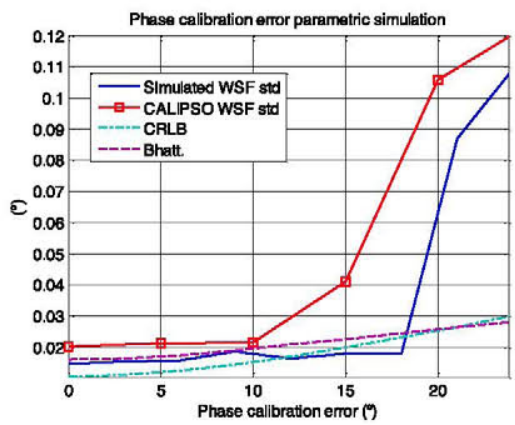

Fig. 5. Calipso acquisition in the presence of phase calibration errors.

$g(\Delta p, \theta, \varphi)$ is a function of the DoA of the satellite, derived from the spherical coordinate frame, and the Cartesian coordinates of the array elements. However, the analog components of the array will have additional phase changes with frequency, which may even be nonlinear for sufficiently large values. Since phase offsets from the probe signal are subtracted from those obtained with the GEO beacon, a linear dependence can be assumed for analysis purposes, though. In this case, the following restriction results:

$$
\Delta f \ll \frac{\Delta \phi}{K} .
$$

$K$ is a parameter representing the aforementioned frequency linear dependence. For example, for frequency offsets of $10 \mathrm{MHz}$ between the signals from the GEO and the desired satellite, the phase dependence parameter $\mathrm{K}$ should be below $0.18^{\circ} \mathrm{MHz}$ in order to obtain a residual phase error one order of magnitude below the threshold. As commented, the probe technique has proven to be effective in SARAS prototype, which implies that the $K$ parameter is low enough for the considered frequency offsets, which are limited to operating band of SARAS system, between 2.2 and $2.3 \mathrm{GHz}$.

\section{Test Campaigns and Conclusion}

An integral calibration scheme has been presented, which combines a variety of techniques and calibration sources in order to obtain the required data for the DoA estimation stage. The system has been successfully tested with a variety of real satellite signals (Cryosat-2, INTEGRAL, XMM-Newton, Calipso, etc.), reaching a Technology Readiness Level of 6. Fig. 5 shows actual results using the DoA estimation algorithm, weighted subspace fitting (WSF) [9], with Calipso satellite, comparing them to simulation data.

The test evaluates the resilience of WSF against phase calibration errors. Simulation results confirm the use of the $18^{\circ}$ threshold that was previously commented. Calipso results show good agreements, with a slightly smaller threshold, between $15^{\circ}$ and $18^{\circ}$. Fig. 5 also shows CRLB and the Bhattacharyya bound for the standard deviation of the estimators [10]. The fact that simulated values are below these bounds for large calibration errors is due to the need of more Monte Carlo iterations to achieve convergence.

Tracking tests performed with a single spacecraft over an 8-h time period have shown that, with the proposed calibration scheme, a system reliability above $99.7 \%$ can be obtained for C/No during acquisition above $17.2 \mathrm{dBHz}$. On the other hand, the first tests that were carried out using only offline calibration models showed that phase instabilities, mainly due to the analog frequency mixers, caused unacceptable DoA errors even with time lapses between calibration and acquisition as small as $5 \mathrm{~min}$.

Thus, the proposed calibration scheme presents clear advantages over conventional calibration techniques, limited to a single stage. With respect to purely parametric calibration, the scheme is more robust against modeling and dynamic errors. Indeed, even the most sophisticated array model will not capture all the possible extraneous variables that can affect the phase response of the array. On the other hand, the inclusion of a parametric calibration for position estimation provides a suitable starting point and eliminates the spurious phase offsets of GEO satellite signals due to the topology of the array. Furthermore, it permits to perform offline calibration using beacons that originate from other directions besides broadside. Finally, the combination of offline and online stages guarantees that the subreflector probe can be used, while eliminating the phase differences with respect to a satellite calibrator and keeping multipath degradation below acceptable thresholds.

Precisely, the use of a collimation tower instead of a GEO satellite beacon is precluded for the moment, due to the influence of multipath. However, if multipath can be precompensated, this solution would eliminate the influence of frequency-dependent errors, as the collimation tower has full flexibility regarding the operating frequency. This is proposed as a potential improvement of the calibration scheme in future implementations of SARAS.

\section{REFERENCES}

[1] I. Garcia-Rojo, A. Anton, A. Giron, and E. Morales, "SARAS: New concept of acquisition aid system for the fast localization and tracking of satellites and launchers," in Proc. 65th Int. Astron. Congr., 2014.

[2] A. Anton, I. Garcia-Rojo, A. Giron, and E. Morales, "Advanced array signal processing algorithms for DoA estimation of satellites and launchers," in Proc. 7th Adv. Satell. Multimedia Syst. Conf. \& 13th Signal Process. Space Commun. Workshop, 2014, pp. 78-85.

[3] T. Svoboda. (2003). "Quick guide to multi-camera self-calibration," [Online]. Available: http://cmp.felk.cvut.cz/svoboda/SelfCal/Publ/ selfcal.pdf

[4] R. S. Pappa, L. R. Giersch, and J. M. Quagliaroli, "Photogrammetry of a $5 \mathrm{~m}$ inflatable space antenna with consumer-grade digital cameras," Exp. Tech., vol. 25, no. 4, pp. 21-29, Jul. 2001.

[5] N. U. Shankar et al., "Photogrammetric measurements of a 12-metre preloaded parabolic dish antenna," in Proc. Nat. Workshop Design Antenna Radar Syst., May 2009.

[6] D. McWatters, A. Freedman, T. Michel, and V. Cable, "Antenna autocalibration and metrology approach for the AFRL/JPL space based radar," in Proc. IEEE Radar Conf., 2004, pp. 21-26.

[7] K.-M. Cheung, "Eigen theory for optimal signal combining: A unified approach," Telecommun. Data Acquisition Prog. Rep., vol. 126, pp. 1-9, Apr. 1996.

[8] C. L. Thornton and J. S. Border, Radiometric Tracking Techniques for Deep-Space Navigation. New York, NY, USA: Wiley, 2003.

[9] H. L. Van Trees, Detection, Estimation, and Modulation Theory, Optimum Array Processing, Part IV. New York, NY, USA: Wiley, 2002.

[10] A. K. Bhattacharyya, "On some analogues of the amount of information and their use in statistical estimation," Indian J. Statist., vol. 8, no. 3, pp. 201-218, 1947. 